\title{
Société Française de Physique : évolutions récentes
}

Cet éditorial fait suite à celui de mars 2012 où se trouvaient exposés les éléments de base de notre politique. Nous allons donc résumer ici les actions menées depuis cette date, dont les principaux objectifs sont d'améliorer notre communication et de valoriser nos activités, tant au niveau local que national.

Je commencerai par une excellente nouvelle : le nouveau site web est maintenant opérationnel (voir p. 24) et semble donner satisfaction aux utilisateurs. Je tiens d'abord à remercier les deux personnes qui se sont impliquées dans sa réalisation, à savoir Raphaël Candelier et Paul-Éric Pottie. Ce site est un outil essentiel de diffusion de I'information et de communication interne et externe. Nous envisageons de lui apporter des améliorations avec l'aide éventuelle d'un professionnel. II importe aussi de le faire vivre : I'apport de chacun d'entre vous sera très apprécié.

Parmi nos objectifs figurait un couplage accru avec les organismes de recherche et les universités. De ce point de vue, nous avons eu des contacts très étroits avec I'Institut de Physique (INP) du CNRS. En particulier, nous avons pu participer au conseil scientifique, à la réunion des directeurs d'unités et aux journées des entrants pour y exposer la stratégie de la SFP. Nous discutons actuellement d'une politique éditoriale commune concernant notre revue Reflets de la physique et les Images de la physique du CNRS. Enfin, nous allons intensifier notre collaboration en ce qui concerne l'alimentation réciproque de nos sites web. Il va de soi qu'une telle politique devra être élargie à d'autres instituts du CNRS, à d'autres organismes de recherche ainsi qu'aux universités, ce qui est déjà partiellement le cas. Toutefois, ceci demande une grande disponibilité, et toute bonne volonté désirant agir en ce sens est instamment priée de se faire connaître.

À mon sens, une des actions les plus importantes en faveur de l'accroissement du nombre d'adhérents à la SFP est la sensibilisation des directeurs d'unités à ce problème. Une première démarche s'est faite lors de la réunion des directeurs d'unités INP, où j'ai pu intervenir en ce sens.

Avec l'accord de Jean-François Pinton, directeur de l'INP, et l'aide d'Amand George, membre du bureau de la SFP, nous avons aussi pu réaliser une évaluation du pourcentage d'adhérents de chacune des unités. Les résultats en seront communiqués à l'ensemble des directeurs et publiés sur le site de la SFP. On peut déjà en tirer une conclusion : la marge de progression est très importante, et il est essentiel que tous se sentent concernés par ce problème typiquement français, mais très handicapant pour la promotion de notre domaine.

Je voudrais maintenant mettre en relief une réalisation importante de notre Commission Énergie-Environnement, à savoir la rédaction d'un livre intitulé La situation énergétique en France et dans le monde, publié par EDP Sciences (voir la présentation dans Reflets $n^{\circ} 29$, p. 34). Cet ouvrage est extrêmement bien fait et fournit une information factuelle, sous une forme accessible à tous. Il est l'exemple d'un des vecteurs par lesquels la SFP peut transmettre l'opinion des scientifiques sur de grands problèmes de société. Ceci m'amène à évoquer notre coopération avec EDP Sciences que nous comptons poursuivre, voire renforcer, en particulier sur les problèmes actuels de publications (Open Access Gold, voir I'article pp. 28-31).

Il est important de chercher à résoudre le problème de la multiplicité des sociétés savantes. De ce point de vue nous continuons à nous impliquer dans la F2S (Fédération Française de Sociétés Scientifiques), qui regroupe trois (et bientôt quatre) sociétés savantes sur des projets ciblés, telle l'action auprès des lycéens. Nous travaillons à étendre ce type de contacts pour une meilleure promotion de la science et de la technologie en France.

Pour terminer, je voudrais mentionner l'échec de la lettre aux candidats à l'élection présidentielle publiée dans le numéro précédent. En effet, seuls deux candidats (parmi les plus mal placés) ont répondu. Peut-être que les questions posées étaient trop précises, peut-être que la recherche est devenue un objectif mineur, peut-être ne sommes-nous pas assez puissants...? Chacun pourra essayer d'apporter sa propre réponse.

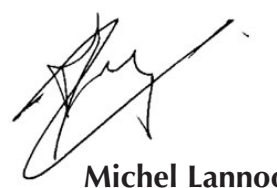

Président de la Société Francaise de Physique (SFP) Directeur de Recherche émérite au CNRS 